\title{
Nonradiative Energy Transfer and Photocurrent Enhancements in Hybrid Quantum Dot-MoS2 Devices
}

\author{
John J. Gough ${ }^{1}$, Niall McEvoy ${ }^{2,3}$, Maria O'Brien ${ }^{2,3}$, Alan P. Bell ${ }^{2}$, John McManus ${ }^{2,3}$, David McCloskey ${ }^{1,3}$, \\ John B. Boland, ${ }^{1,3}$ Jonathan N. Coleman ${ }^{1,3}$, Georg S. Duesberg, ${ }^{2,3,4}$, and A. Louise Bradley ${ }^{1}$ \\ ${ }^{1}$ School of Physics and CRANN, Trinity College Dublin, Dublin 2, Ireland \\ ${ }^{2}$ School of Chemistry and CRANN, Trinity College Dublin, Dublin 2, Ireland \\ ${ }^{3} A M B E R$ Centre, Trinity College Dublin, Dublin 2, Ireland \\ ${ }^{4}$ Institute of Physics, EIT 2, Faculty of Electrical Engineering Information Technology, Universität der \\ Bundeswehr München, Werner-Heisenberg-Weg 39, 85577 Neubiberg, Germany \\ Tel: (3531) 896 3595, Fax: (3531) 896 2711, e-mail: bradlel@tcd.ie
}

\begin{abstract}
The energy transfer mechanism leading to highly efficient nonradiative energy transfer (NRET) from quantum dots (QDs) to monolayer $\mathrm{MoS}_{2}$ devices has been investigated via a spectral dependence study. The spectral dependence of the NRET from three spectrally separated QD ensembles to monolayer $\mathrm{MoS}_{2}$ devices reveals that the trends in NRET rates follow the trends in the spectral overlap between the QD emission and $\mathrm{MoS}_{2}$ absorption spectra, thus verifying that the mechanism for the energy transfer is Förster-type NRET. Furthermore, the dependence of the photocurrent enhancement on the $\mathrm{MoS}_{2}$ film properties is also explored.
\end{abstract}

Keywords: nonradiative energy transfer, quantum dots, 2D materials, photocurrent, photoresponsivity.

\section{INTRODUCTION}

In recent years there has been a significant focus on research into $2 \mathrm{D}$ materials due to their unique optical and electrical properties. It was the discovery and isolation of graphene that initially sparked interest in $2 \mathrm{D}$ materials [1]. Graphene, however, lacks a direct bandgap which is a desirable property when considering optoelectronic devices. Transition metal dichalcogenides (TMDs) such as $\mathrm{MoS}_{2}, \mathrm{WS}_{2}, \mathrm{WSe}_{2}$ and $\mathrm{MoSe}_{2}$ can also be isolated into monolayers and have the advantage of a transition to direct bandgap semiconductors at monolayer thicknesses [2]. The presence of a direct bandgap at monolayer thickness gives these TMDs the edge over graphene in terms of optoelectronic devices. However, when considering an optoelectronic device architecture that consists of monolayer materials, the low levels of optical absorption must be taken into account. The inclusion of a sensitizing layer is beneficial in terms of enhancing the optical absorption in the hybrid system and must facilitate the efficient transfer of energy from the sensitizer to the monolayer material. One particular mechanism of energy transfer from the sensitizing species to the active material is Förster-type nonradiative energy transfer (NRET). This has been proven to be a highly efficient process in donor-acceptor pairs of quantum dots (QDs) and monolayer TMDs [3][5]. It has also been revealed that, in contrast to graphene, the NRET rate between QDs and $\mathrm{MoS}_{2}$ decreases as the layer number of the material increases. This behaviour is a consequence of the strong increase in dielectric screening with the addition of each $\mathrm{MoS}_{2}$ layer [4]. The incorporation of a sensitizing species on $\mathrm{MoS}_{2}$ photodetector devices has been shown to substantially increase the photocurrent and photoresponsivity of the devices [5]-[7]. In this contribution it is confirmed that the energy transfer mechanism in QD-sensitised $\mathrm{MoS}_{2}$ devices is Förster-type NRET. This has been probed via a spectral dependence study of NRET to monolayer $\mathrm{MoS}_{2}$ device using three QD ensembles with emission wavelengths spanning different regions of the visible spectrum. The effect of the CVD-grown $\mathrm{MoS}_{2}$ film properties on the photocurrent enhancements is also reported.

\section{EXPERIMENTAL METHODS}

$\mathrm{MoS}_{2}$ of various film properties and thickness was grown on a $\mathrm{Si} / \mathrm{SiO}_{2}$ substrate using a large area coverage chemical vapor deposition (CVD) growth technique [8].

Alloyed core/shell CdSeS/ZnS QDs were purchased from Sigma-Aldrich in toluene $(1 \mathrm{mg} / \mathrm{mL})$. In order to form thin film layers the QDs were dispersed in $0.1 \% \mathrm{wt}$. PMMA in toluene. The QD/PMMA solutions were prepared by dispersing $12.5 \mu \mathrm{L}$ of stock QD solution in $500 \mu \mathrm{L}$ of $0.1 \%$ wt. PMMA in toluene. The dispersions were sonicated for $\sim 20 \mathrm{~s}$ to ensure even distribution of the QDs in the PMMA matrix. The alloyed QDs with peak emission wavelengths of $450 \mathrm{~nm}, 530 \mathrm{~nm}$, and $630 \mathrm{~nm}$ have an average diameter of $(6 \pm 0.8) \mathrm{nm}$.

The $\mathrm{MoS}_{2}$ devices were fabricated via the patterning of a PMMA e-beam resist using electron beam lithography. The contact pads and electrodes consisting of Ti/Au $(5 \mathrm{~nm} / 45 \mathrm{~nm})$ were deposited using an e-beam evaporation technique. For the spectral dependence study, the device samples were grown on different $\mathrm{Si} / \mathrm{SiO}_{2}$ chips for each of the QD ensembles during a single growth process. For the $\mathrm{MoS}_{2}$ film properties study, all devices were grown on the same $\mathrm{Si} / \mathrm{SiO}_{2}$ chip in a single growth process. The hybrid QD-MoS $\mathrm{M}_{2}$ devices were fabricated by spin coating an ultrathin layer of QDs in PMMA on top of the $\mathrm{MoS}_{2}$ devices to obtain monolayer coverage of QDs. 
Time-resolved PL (TRPL) measurements were performed using an excitation with 90 ps pulses at a wavelength of $405 \mathrm{~nm}$ and a repetition rate of $10 \mathrm{MHz}$. The sample was excited through a $40 \times$ objective with an NA of 0.65 and a spot size of $\sim 430 \mathrm{~nm}$. The PL was collected through the same objective.

The photocurrent measurements were performed using a variable power laser diode with an excitation wavelength of $405 \mathrm{~nm}$. The active region in the device was excited through a 10× objective with an NA of 0.25 and a spot size of $\sim 3 \mu \mathrm{m}$. A Keithley 2400 source meter was used to provide a bias and measure the current flowing through the devices.

\section{RESULTS AND DISCUSSION}

The extinction spectrum of monolayer $\mathrm{MoS}_{2}$ and the extinction spectra of each of the QD ensembles are shown in Fig. 1(a), along with the normalized PL spectra of the QDs. There is good overlap between the $630 \mathrm{~nm}$ QDs emission spectrum and the ' $\mathrm{B}$ ' exciton in the $\mathrm{MoS}_{2}$ extinction spectrum. The emission spectrum of the $450 \mathrm{~nm}$ QDs also overlaps quite well with the high energy ' $C$ ' exciton in the $\mathrm{MoS}_{2}$ extinction spectrum, while there is no overlap between the emission spectrum of the $530 \mathrm{~nm}$ QDs with any of the $\mathrm{MoS}_{2}$ exciton peaks. A schematic representation of the hybrid devices is given in Fig. 1(b).

(a)

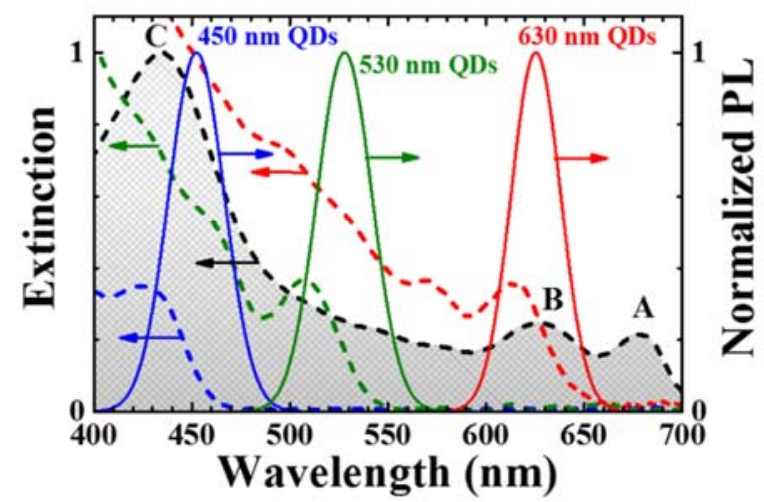

(b)

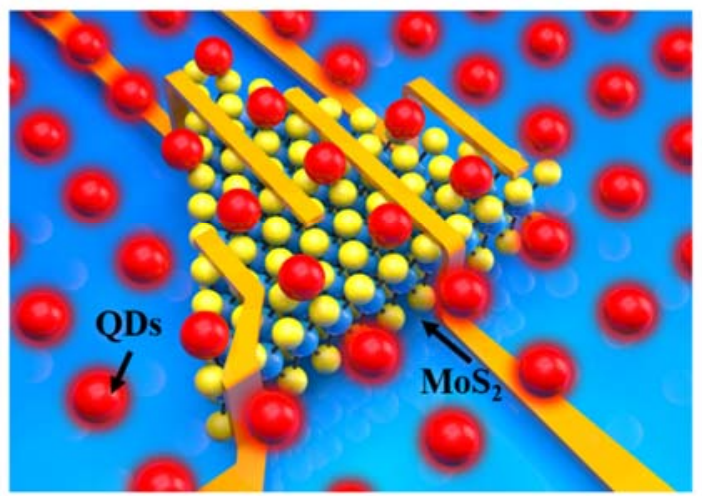

Figure 1: (a) Extinction spectra (left axis) of monolayer $\mathrm{MoS}_{2}$ (black dash), $450 \mathrm{~nm}$ QDs (blue dash), $530 \mathrm{~nm}$ QDs (green dash), and $630 \mathrm{~nm}$ QDs (red dash), and normalized PL spectra (right axis) of $450 \mathrm{~nm}$ QDs (blue solid), $530 \mathrm{~nm}$ QDs (green solid), and $630 \mathrm{~nm}$ QDs (red solid). The QD extinction spectra in (a) have been scaled to give the same intensity at the first excitonic peak. (b) Schematic representation of the hybrid QD-MoS devices.

TRPL measurements were performed to measure the lifetime of the QDs on the devices, $\tau_{Q D-M o S_{2}}$, and the lifetime of the QDs off the devices, $\tau_{Q D}$. The PL decays and lifetimes of the $450 \mathrm{~nm}$ QDs (blue), $530 \mathrm{~nm}$ (green), and $630 \mathrm{~nm}$ QDs (red) are shown in Fig. 2, panels (a-c), respectively. The black curves in Fig. 2(a-c) are the PL decays of the QDs on the $\mathrm{MoS}_{2}$ devices. There is a significant reduction in the lifetime for each QD ensemble on the $\mathrm{MoS}_{2}$ devices. The NRET efficiencies, given by $\eta_{N R E T}=1-\left(\tau_{Q D-M o S_{2}} / \tau_{Q D}\right)$, are $(94 \pm 7) \%,(92 \pm 4) \%$, and $(96 \pm 6) \%$, for the $450 \mathrm{~nm}, 530 \mathrm{~nm}$, and $630 \mathrm{~nm}$ QDs, respectively.

The measured photocurrents, $I^{P h}$, at a bias of $+1 \mathrm{~V}$ for each of the devices before and after adding the QD sensitizing layer, as a function of optical excitation power, $P_{E x}$, are shown in Fig. 2(d-f). There is a significant increase of the photocurrent of each device after the addition of the QD sensitizing layers. It should be noted that the photocurrent as a function of optical excitation power has the same trend before and after adding the sensitizing layers. Regions corresponding to sub-linear, linear, and super-linear dependence of the photocurrent on the optical excitation power are observed. The sub-linear and linear dependence of photocurrent in $\mathrm{MoS}_{2}$ devices is commonly observed, however, the super-linear dependence is less reported and is believed to be due to the presence of a variety of recombination centres in the $\mathrm{MoS}_{2}$ associated with surface imperfections such as surface defects, dangling bonds, and edge defects at grain boundaries [9].

An average $\mathrm{MoS}_{2}$ photocurrent enhancement, $E_{P h}^{A v g}$, is calculated from the low power data, where the photocurrent exhibits a dominant $I^{P h}=A P_{E x}^{0.5}$ dependence. This allows for comparison between the photocurrent values and the NRET efficiencies, as the TRPL measurements were performed at low excitation powers $(0.2 \mu \mathrm{W})$. The average photocurrent enhancement, $E_{P h}^{A v g}=\left(A_{M o S_{2}-Q D} / A_{M o S_{2}}-1\right)$, gives values of $2.9 \pm 0.5,1.5 \pm 0.1$, and $6 \pm 1$ for devices with the $450 \mathrm{~nm}, 530 \mathrm{~nm}$, and $630 \mathrm{~nm}$ QD sensitizing layers, respectively. There is good agreement between the trends of NRET efficiency and the average $\mathrm{MoS}_{2}$ photocurrent enhancement, indicating that the efficiency of the NRET is driving the enhancements in the $\mathrm{MoS}_{2}$ devices. Similarly, a comparison of the NRET rates calculated from the lifetime data and and the spectral overlap integral reveal almost identical trends, thus confirming that the energy transfer process is that of Förster-type NRET. 
(a)

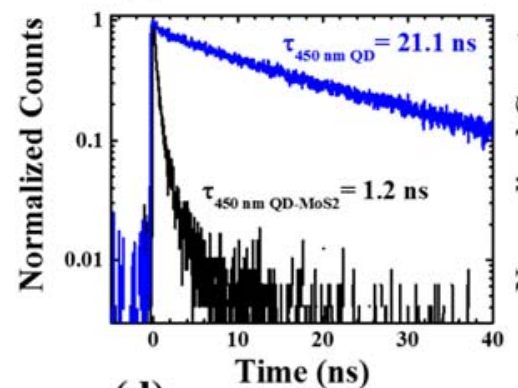

(d)

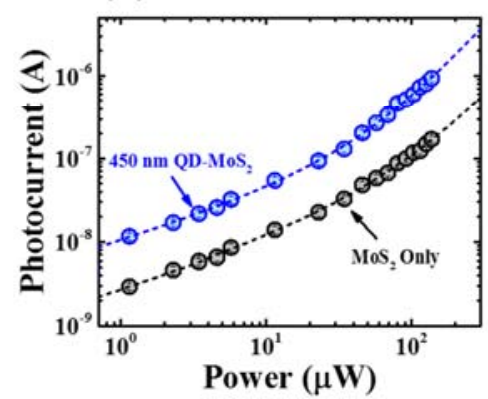

(b)

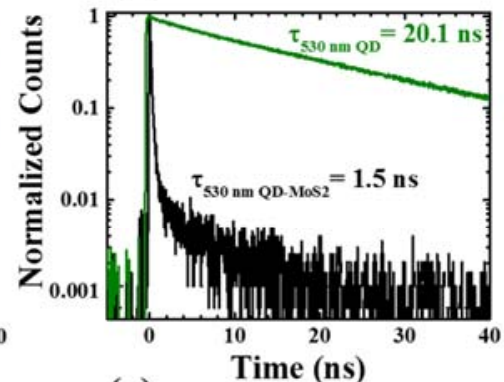

(e)

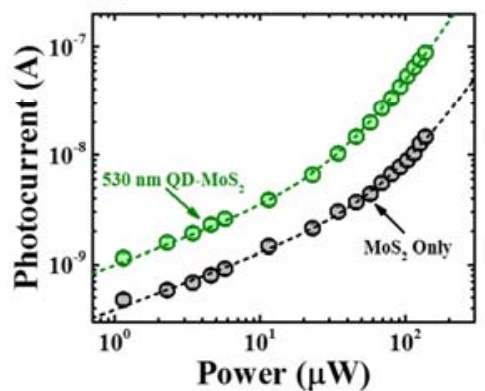

(c)

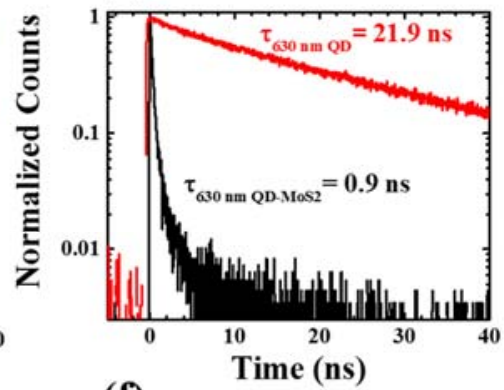

(f)

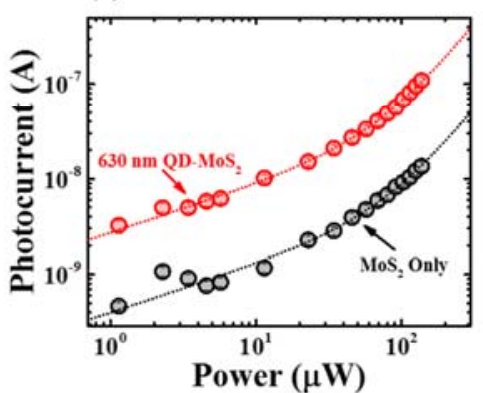

Figure 2: PL decays of (a) $450 \mathrm{~nm}$ QDs only (blue) and on the MoS 2 device (black), (b) $530 \mathrm{~nm}$ QDs only (green) and on the $\mathrm{MoS}_{2}$ device, and (c) $630 \mathrm{~nm}$ QDs only (red) and on the $\mathrm{MoS}_{2}$ device. (d-f) Photocurrent measured from the $\mathrm{MoS}_{2}$ devices before (black) and after adding the QD sensitizing layers.

As the $630 \mathrm{~nm}$ QDs show the largest NRET efficiency and lead to the largest photocurrent enhancements in the hybrid devices, these QDs were chosen to study of the dependence of the photocurrent enhancements on the $\mathrm{MoS}_{2}$ material layer properties. These $\mathrm{MoS}_{2}$ devices consisted of pristine monolayer (Mono), mixed monolayer/bilayer with a greater proportion of monolayer to bilayer material (Mono/Bi), mixed monolayer/bilayer with a greater proportion of bilayer to monolayer material (Bi/Mono), and polycrystalline bilayer (Poly Bi). It can be seen from the photocurrent plots, presented in Fig. 3(a-d), that the largest enhancements are observed in the monolayer material ( $\sim 14$-fold), with moderate enhancements in the devices consisting of mixed layer material ( $\sim 2$-fold), and no enhancement in the polycrystalline bilayer devices.

(a)

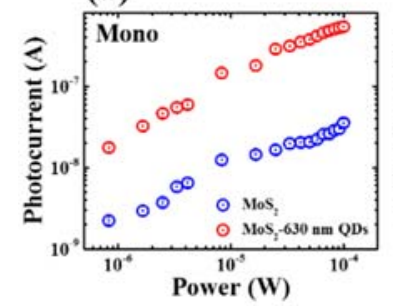

(e)

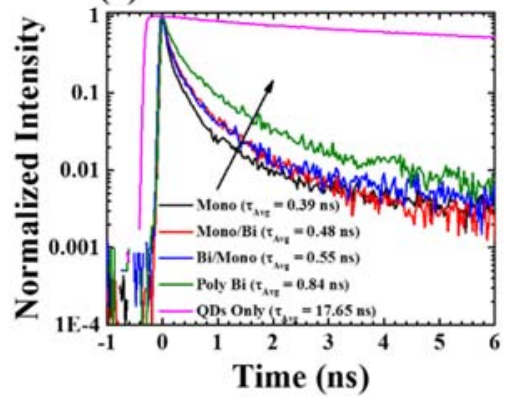

(c)

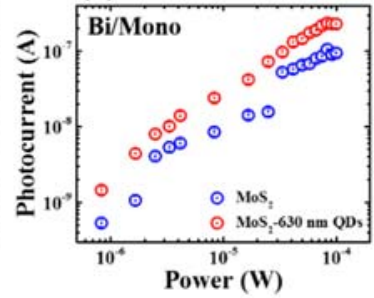

(f)

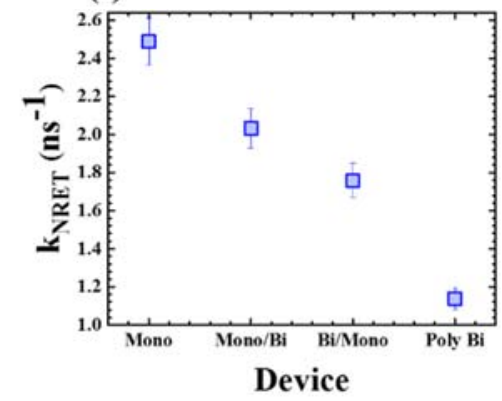

Figure 3: (a-d) Photocurrent as a function of optical excitation power for the $\mathrm{MoS}_{2}$ devices before (blue) and after (red) adding the QD sensitizing layer. (e) PL decays for the $630 \mathrm{~nm}$ QDs on each of the $\mathrm{MoS}_{2}$ devices. (f) NRET rates for the QDs on the $\mathrm{MoS}_{2}$ devices. 
TRPL measurements were also performed on the QDs on each of these devices, and the PL decays are presented in Fig. 3(e). The shortest QD PL lifetime is measured on the pristine monolayer $\mathrm{MoS}_{2}$ device and the PL lifetime of the QDs increases as the proportion of $\mathrm{MoS}_{2}$ in the device channel transitions from pristine monolayer to polycrystalline bilayer, due to the increase in dielectric screening. The NRET rate, $k_{N R E T}$, can be calculated from the QD lifetimes as $k_{N R E T}=\tau_{Q D-M o S_{2}}^{-1}-\tau_{Q D}^{-1}$. The NRET rates for the four devices, presented in Fig. 3(f), show a more gradual decrease than is observed for the photocurrent enhancement. However, the largest and smallest NRET rates are found for the monolayer and polycrystalline bilayer $\mathrm{MoS}_{2}$ devices, respectively. This discrepancy between trend of the photocurrent enhancements and the NRET rates is due to the fact that the photocurrent enhancements rely on the transit of charge carriers across the device channel, which can be obstructed by nucleation points and grain boundaries in the $\mathrm{MoS}_{2}$, while NRET, which is the nonradiative transfer of the QD exciton energy through dipole-dipole coupling, occurs directly between the QDs and the $\mathrm{MoS}_{2}$ due to the Coulomb interaction between confined excitons in both materials.

\section{CONCLUSIONS}

In summary, by comparing the NRET rates and the spectral overlap for three hybrid devices consisting of different QD ensembles, we have shown that the mechanism for NRET in hybrid QD-MoS device architectures is that of Förster-type NRET. It has also been demonstrated that the photocurrent enhancements in monolayer $\mathrm{MoS}_{2}$ devices are driven by the NRET efficiency from these alloyed QDs. The photocurrent enhancements in the hybrid QD$\mathrm{MoS}_{2}$ devices are shown to be strongly dependent on the $\mathrm{MoS}_{2}$ material properties. Despite the presence of highly efficient NRET from the QDs for all devices, the photocurrent enhancements are significantly higher for pristine monolayer $\mathrm{MoS}_{2}$. In the case of mixed monolayer/bilayer and polycrystalline $\mathrm{MoS}_{2}$ the presence of nucleation points and defect sites in the $\mathrm{MoS}_{2}$ leads to scattering of charge carriers and subsequently, reduced mobility.

\section{ACKNOWLEDGEMENTS}

This work was supported by the Irish Research Council (IRC), Grant No. GOIPG/2013/680 and Science Foundation Ireland (SFI), Grant Nos. 10/IN.1/12975 and 16/IA/4550.

\section{REFERENCES}

[1] K. S. Novoselov et al., "Electric field effect in atomically thin carbon films.," Science (80), vol. 306, no. 5696, pp. 666-669, 2004.

[2] Y. Li et al., "Measurement of the optical dielectric function of monolayer transition-metal dichalcogenides: $\mathrm{MoS}_{2}, \mathrm{MoSe}_{2}, \mathrm{WS}_{2}$, and WSe 2 ," Phys. Rev. B, vol. 90, no. 20, p. 205422, 2014.

[3] K. M. Goodfellow et al., "Distance-dependent energy transfer between CdSe/CdS quantum dots and a two-dimensional semiconductor," Appl. Phys. Lett., vol. 108, no. 2, p. 21101, 2016.

[4] A. Raja et al., "Energy transfer from quantum dots to graphene and $\mathrm{MoS}_{2}$ : The role of absorption and screening in two-dimensional materials.," Nano Lett., vol. 16, no. 4, pp. 2328-2333, 2016.

[5] J. J. Gough et al., "Dependence of Photocurrent Enhancements in Quantum Dot (QD)-Sensitized $\mathrm{MoS}_{2}$ Devices on $\mathrm{MoS}_{2}$ Film Properties," Adv. Funct. Mater., vol. 1706149, p. 1706149, 2018.

[6] S. H. Yu et al., "Dye-sensitized $\mathrm{MoS}_{2}$ photodetector with enhanced spectral photoresponse," ACS Nano, vol. 8, no. 8, pp. 8285-8291, 2014.

[7] D. Kufer, I. Nikitskiy, T. Lasanta, G. Navickaite, F. H. L. Koppens, and G. Konstantatos, "Hybrid 2D-0D $\mathrm{MoS}_{2}-\mathrm{PbS}$ quantum dot photodetectors.," Adv. Mater., vol. 27, no. 1, pp. 176-80, 2015.

[8] M. O’Brien et al., "Transition Metal Dichalcogenide Growth via Close Proximity Precursor Supply," Sci. Rep., vol. 4, p. 7374, 2014.

[9] V. Klee et al., "Superlinear Composition-Dependent Photocurrent in CVD-Grown Monolayer $\operatorname{MoS}_{2}(1-$ x)Se 2 X Alloy Devices," Nano Lett., vol. 15, no. 4, pp. 2612-2619, 2015. 